\title{
Combating COVID-19 with Mesenchymal Stem Cell therapy
}

Keshav Rajarshi ${ }^{1}$, Aroni Chatterjee ${ }^{2}$ and Shashikant Ray $^{3 *}$

${ }^{1}$ School of Community Science and Technology (SOCSAT) Indian Institute of Engineering Scince and Technology (IIEST), Shibpur, Howrah, West Bengal-711103, India

${ }^{2}$ Indian Council of Medical Research (ICMR) - Virus Research Laboratory, NICED, Kolkata, India

${ }^{3}$ Department of Biotechnology, Mahatma Gandhi Central University Motihari-845401, India

*Correspondence may be addressed to this author

3*. Shashikant Ray, Assistant Professor, Department of Biotechnology, Mahatma Gandhi Central University Motihari-845401, India, E-mail: shashikantray@mgcub.ac.in

Keywords: COVID-19, Mesenchymal Stem Cells, SARS-CoV-2, Immunomodulatory and Cytokines 


\begin{abstract}
The world is currently facing one of its deadliest nightmares, the rise of a global pandemic called COVID-19. The disease is caused by a positive stranded RNA virus called SARSCoV-2. The virus mainly targets the pulmonary epithelial cells as it's initial site of infection by letting its surface spike protein interact and bind to the host ACE2 receptor. The internalization and gradual replication of the virus results in an exaggerated immune response triggering release of many pro-inflammatory cytokines and chemokines. This immune storm is responsible for multiple health hazards in the host ultimately leading to multiple organ failure. Mesenchymal stem cell therapy offers a promising approach towards mitigating the delirious effects of the infection in the COVID-19 patients. This therapy has shown to reduce the expression of pro-inflammatory cytokines as well as repair of damaged tissues in COVID-19 patients. This review has been organized to put forward all the positive aruments and implications in support of mesenchymal stem cell therapy as a necessary approach for treating COVID-19 patients.
\end{abstract}

\title{
1. Introduction
}

The end of the year 2019 marked the beginning of a new challenge for humanity when several cases of severe respiratory ailments were reported in the city of Wuhan, Hubei province, China. The cases were earlier confused with regular flu and thought to be caused by the normal seasonal influenza virus. The accurate prognosis of the illness was very difficult to make in the beginning but was simulataneously identified to be a virus borne disesase. Due to the increasing severity in the following days, on January 1, the virus was declared novel. Upon the complete phylogenetic analysis of the viral genome consisting of 29,903 nucleotides, it was found that this novel coronavirus had $89.1 \%$ similar nucleotides to a class of Severe Acute Respiratory Syndrome (SARS) - like coronavirus. This novel virus belonged to the genus Betacoronavirus having the subgenus Sarbecovirus [1]. The novel coronavirus was earlier known to be found in Chinese bats [2]. WHO has assigned a brief name to the virus, SARS-CoV-2 and COVID-19 is the name assigned to the SARS-CoV-2 associated disease. 
Till date no dedicated therapeutic or vaccination strategies have been implemented or confirmed to prevent COVID-19. Accessory therapeutic manueveurs including corticosteroid mediated inflammation reduction, convalescent plasma therapy, antibiotics for treatment of secondary bacterial sepsis and non-specific antivirals, etc., do not show much effectivity in severe cases of COVID-19. The main reason behind the failure of these therapies is the cytokine storm in the lungs generated by the virus. In the computed tomography scans, these cytokine storms (an augmented immune response in the body towards any external stimulus) appear as inflammatory lesions with ground-glass opacity $[3,4]$.

Most of the gravely sick and deceased patients do not develop any severe clinical manifestations in the earlier stages of COVID-19. The commonly reported symptoms are cough, throat pain, mild/high fever, muscle soreness, or body ache. A sudden deterioration in the health condition of the patients are seen in the later stages of diseases progression. Rapid failure of multiple organs and ARDS (Acute Respiratory Distress Syndrome) results in death within a very short period of time. Cytokine storm has been indicated as the presumable causal factor for ARDS and multiple organ failure [5, 6] (Figure 1).

WHO has approximated the case fatality rate (CFR) of COVID-19 as ranging from $0.3-1 \%$, higher than that of influenza A which has a CFR of $0.1 \%$. Various epidemiological studies conducted in countries implementing COVID-19 mitigation strategies has reported that around $80 \%$ of patients suffering from COVID-19 had symptomless or mild disease. $14 \%$ of the patients had severe ailments, and $6 \%$ of them were in critical condition [7] (Figure 2) .

\subsection{Pathogenesis of SARS-CoV-2}

SARS-CoV-2 recognizes the angiotensin I converting enzyme 2 (ACE2) receptor by its spike glycoprotein (SARS-CoV-2S), which is a class I fusion protein. The virus is consecutively fused with the host cells. S1 subunit, via its receptor-binding domain, mediates the attachment of the spike protein. The protein changes its conformation after binding with the receptor. S2 domain of the spike protein is responsible for membrane fusion and internalization of the virus [8]. The host cell entry and spread are facilitated by priming of the spike protein of the virus by cellular transmembrane protease, serine 2 (TMPRSS2) [9-11] .

In the lungs, the ACE2 receptor and TMPRSS2 is commonly expressed in alveolar type II cells along with capillary endothelial cells. Therefore the characteristic symptom of SARS$\mathrm{CoV}-2$ infection is the severe respiratory ailment. This viral infection within the lungs results 
in a cytokine storm, thus elevating the level of many pro-inflammatory cytokines. The increased level of these cytokines leads to oedema, dysfunction in exchange of air, acute respiratory distress, and various other secondary infections, which may eventually cause death [9-12].

\subsection{Why Mesenchymal Stem Cells?}

Presently, cell-based therapy, and above all, stem cell therapy has proven itself to be one of the most promising therapeutic approaches that provide opportunities to treat several diseases that were considered incurable earlier [13]. MSC therapy is preferred over other therapeutic strategies because they are free of ethical and social issues, they have a high proliferation rate and a low invasive nature. Mesenchymal cells can be obtained from various sources, including adipose tissues, dental pulp, bone marrow, umbilical cord, menstrual blood, fetal liver, and Bichat's fat pad. MSCs can also be isolated from various adult tissues such as the infrapatellar fat pad, abdominal fat pad, and tissues associated with neonates such as placenta, Wharton's (gelatinous substance providing insulation and protection to the umbilical cord) jelly, cord blood, and amniotic fluid. These stem cells are multipotent (i.e., having several fates). Storage of mesenchymal cells can be done so that they can be repetitively used for therapeutic purposes as they expand to volume in a suitable and short period of time. So far, the clinical trials of mesenchymal stem cells have not shown any unfavorable reaction towards the allogeneic MSCs. The efficacy and safety of the MSCs have been documented in many clinical trials very well [14].

\subsection{Therapeutic achievements}

Remarkable reversal of severe COVID-19 symptoms even in critical condition were reported in two clinical studies conducted in China. These clinical studies discovered a novel therapeutic strategy as well as existing natural mechanism resisting acute inflammatory pneumonia.

A study was conducted on 7 SARS-CoV-2 positive patients, out of which 4 of them showed severe symptoms, two showing common types of the syndrome, and 1 of them was critically ill [9]. Clinical-grade human MSCs were intravenously administered to each of the patients. The patients were treated with $1 \times 10^{6}$ MSCs per kilogram body weight while their condition was worsening severely. They were observed for a total period of 14 days. Before the infusion of MSCs, all the patients had high fever, reduced oxygen saturation, dyspnea 
(shortness of breath) and pneumonia. The study showed that almost all the symptoms displayed by the patients before infusion, subsided under 2-4 days after they received the infusion. The peripheral lymphocytes increased after the treatment, with a shift towards the regulatory phenotype for both dendritic cells and CD4+T cells. The MSC infusion didn't show any side effects. CT scans of chest revealed that pneumonia infiltration was remarkably reduced. Most of the patients were reported negative for the SARS-CoV-2 nucleic acid test within 7-14 days after the infusion. The most exciting and extraordinary finding of the study was the overall improvement of an elderly patient who was critically ill previously.

Another study involved a critically ill ventilator ridden COVID-19 patient who was administered with human umbilical cord MSC (hUCMSC). This patient was treated with 3 infusions of $5 \times 10^{7} \mathrm{hUCMSC}$ at the interval of 3 days, and the patient was able to walk within 4 days of her second cell infusion. The essential parameters like T-cell counts were restored back to normal levels. The patient displayed no observable side effects [15].

Till date about 17 completed clinical studies along with 70 trials are registered on https://clinicaltrials.gov, demonstrating the immunomodulatory and anti-inflammatory properties of MSCs in treating respiratory ailments. Also, Chinese clinical trial registry site, http://www.chictr.org.cn, has reported registration of 20 clinical trials in this regard.

\section{MSC therapy: analysis of outcomes}

COVID-19 triggers an exaggerated immune reaction in the body by producing large amounts of various inflammatory factors including several cytokines, chemokines and immune reactive cells. It can be hypothesized that the MSC therapy might prevent the triggering of cytokine storm by the activated immune system, and the reparative properties of the stem cells might promote endogenous repair [16]. Mesenchymal stem cells when intravenously injected will lead to some part of the population getting entrapped in the lungs. The pulmonary microenvironment could be recovered with the help of these MSCs, thus protecting the alveolar epithelial cells. Hence, pulmonary fibrosis of the lungs could be prevented, which may lead to curing COVID-19 caused pneumonia. In the multiple disease condition, the immunomodulatory effects will be responsible for improved function after MSC infusion. A variety of paracrine factors are secreted by these cells. These paracrine factors interact with the immune cells, eventually leading to immunomodulation. The vigorous anti-inflammatory activities of MSCs will actually be responsible for improvements after their infusion in COVID-19 patients. 
IUt has been found that after infusion of MSCs in COVID-19 patients, the number of peripheral lymphocytes increased while the levels of C-reactive protein (CRP) decreased. The overactivated cytokine-secreting immune cells dwindled within 6 days in the circulating blood. Furthermore, after the MSC treatment, the population of regulatory dendritic cells $\left(\mathrm{CD} 14^{+} \mathrm{CD} 11 \mathrm{~b}^{\mathrm{mid}}\right)$ increased. A major pro-inflammatory cytokine, TNF- $\alpha$ (Tumour Necrosis Factor), exhibited a decline in its levels in the MSC treated COVID-19 patients, with an elevation in the concentration of IL-10 (Interleukin-10) compared to the patients treated with conventional therapy [9]. Table 1 depicts the various immunomodulatory interventions by the MSCs.

For further elucidation of the underlying mechanisms of MSC-dependent treatment of COVID-19 patients, $10 \times$ RNA-sequencing was done [9], which implicated that MSCs were free from infection with SARS-CoV-2.

\section{Conclusion}

SARS-CoV-2 (novel coronavirus) and SARS-2003, both have a similar mechanism of infection, i.e, binding the spike protein on the viral surface to the ACE2 receptors on the host cell surface. Thus all the tissues and organs expressing the ACE2 receptor are susceptible to the SARS-CoV-2 infection. Since the alveolar epithelial cells have a high propensity of ACE2 receptors, they are the most adversely affected during SARS-CoV-2 infection. The devastating cytokine explosion attributed to the SARS-CoV-2 infection leads to sevre shock, oedema and multiple organ failure. Administering the COVID-19 patients with an infusion of multipotent MSCs can help to combat the COVID-19 as these cells will inhibit the exaggerated immune response and encourage endogenous repair of the lung epithelial cells by improving the microenvironment. The mesenchymal stem cell therapy has not tey shown any adverse side effects on the patient. In this review we have highlighted all the implications associated with MSC therapy application in case of COVID-19 and strongly place our argument in support of this.

\section{Acknowledgements}

KR and SR thanks IIEST Shibpur and Mahatma Gandhi Central University Motihari, Bihar, respectively.

\section{Disclosure statement}

\section{The authors report no conflicts of interest}




\section{Figure 1:}

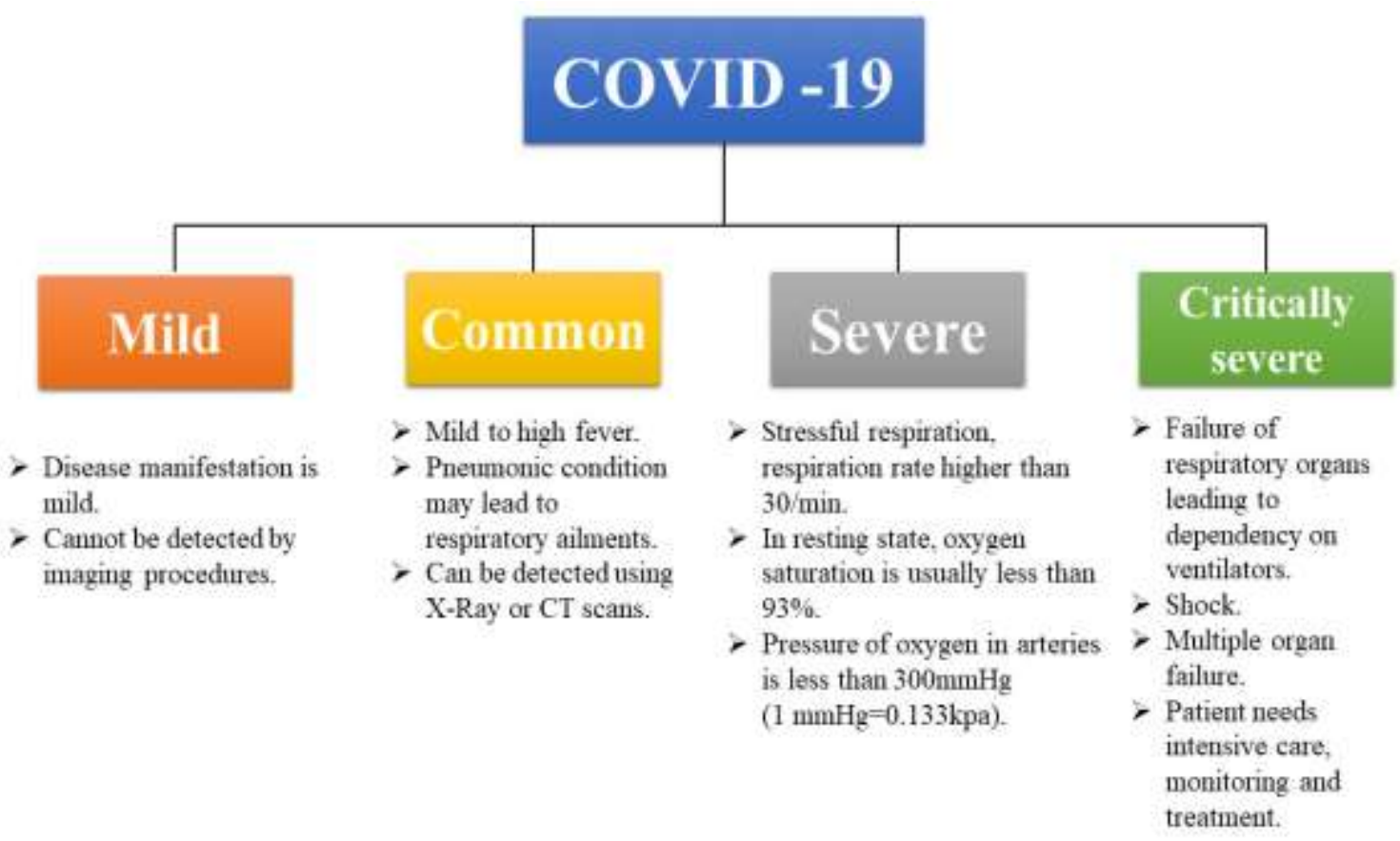

Figure 1: Classification of COVID-19 on clinical basis issued by the National Health Commission of China. 
Figure 2:

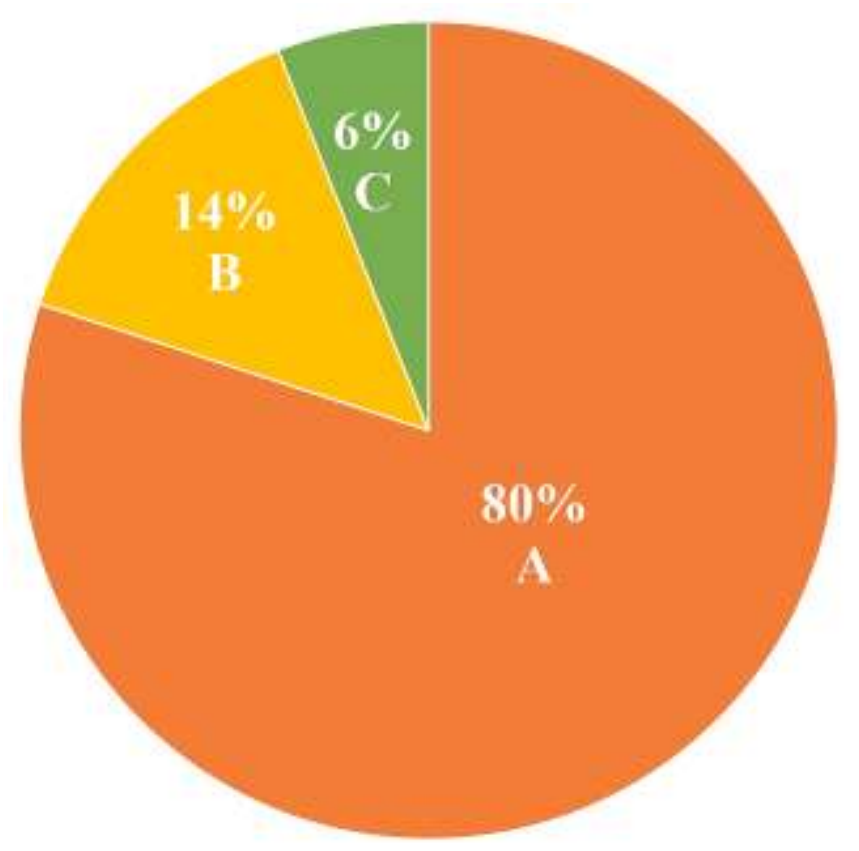

Figure 2: Data obtained from epidemiological studies conducted in various countries reporting the incidence of COVID-19 suggested that (A). $80 \%$ of the patients suffering from COVID-19 were asymptomatic. (B). 14\% of them had severe symptoms, and (C). $6 \%$ of the reported patients were critically ill . 
Table 1:

\begin{tabular}{|c|c|}
\hline Pathological Complications & $\begin{array}{l}\text { MSC therapy intervention } \\
\text { (Immunoregulatory functions) }\end{array}$ \\
\hline 1. Pulmonary inflammatory lesions & $\begin{array}{l}\text { 1. Improvement of lung function and } \\
\text { pulmonary fibrosis by lung } \\
\text { aggregation and protection of } \\
\text { alveolar epithelium }\end{array}$ \\
\hline $\begin{array}{l}\text { 2. Elevation in the levels of aspartic } \\
\text { aminotransferase and creatine kinase } \\
\text { in the serum enzymes as a result of } \\
\text { multiple organ failure }\end{array}$ & $\begin{array}{l}\text { 2. Promotion of endogenous repair in } \\
\text { the tissue/cellular organization by } \\
\text { improvement in the } \\
\text { microenvironment of organization }\end{array}$ \\
\hline $\begin{array}{l}\text { 3. Reduction and overactivation of } \\
\text { CD4 and CD8T cells as a result of } \\
\text { immune cell depletion }\end{array}$ & $\begin{array}{l}\text { 3. Reorganization/rearrangement } \\
\text { functions of immune cell subsets }\end{array}$ \\
\hline $\begin{array}{l}\text { 4. Induction of cytokine storm leading } \\
\text { to enhanced levels of IL-7, IL-2, } \\
\text { IP10 (serum interferon-gamma- } \\
\text { inducible protein-10), MCP-1 } \\
\text { (monocyte chemoattractant protein- } \\
\text { 10), GCSF (granulocyte colony } \\
\text { stimulating factor), MIP-1 } \alpha \\
\text { (macrophage inflammatory protein) } \\
\text { and TNF- } \alpha\end{array}$ & $\begin{array}{l}\text { 4. Regulation of inflammatory } \\
\text { cytokines and inhibition of } \mathrm{T} \text { and } \mathrm{B} \\
\text { lymphocytes }\end{array}$ \\
\hline
\end{tabular}

Table 1: Mesenchymal Stem Cells deprived of ACE2 receptors benefit the patients having SARS-CoV-2 infection via immunomodulatory functions [9]. 


\section{References}

1. Wu, F., Zhao, S., Yu, B., Chen, Y. M., Wang, W., Song, Z. G., Hu, Y., Tao, Z. W., Tian, J. H., Pei, Y. Y., Yuan, M. L., Zhang, Y. L., Dai, F. H., Liu, Y., Wang, Q. M., Zheng, J. J., Xu, L., Holmes, E. C. \& Zhang, Y. Z. (2020) A new coronavirus associated with human respiratory disease in China, Nature. 579, 265-269.

2. Hu, D., Zhu, C., Ai, L., He, T., Wang, Y., Ye, F., Yang, L., Ding, C., Zhu, X., Lv, R., Zhu, J., Hassan, B., Feng, Y., Tan, W. \& Wang, C. (2018) Genomic characterization and infectivity of a novel SARS-like coronavirus in Chinese bats, Emerging microbes \& infections. 7, 154.

3. Metcalfe, S. M. (2020) Mesenchymal stem cells and management of COVID-19 pneumonia, Medicine in drug discovery. 5, 100019.

4. Ye, Q., Wang, B. \& Mao, J. (2020) The pathogenesis and treatment of the 'Cytokine Storm' in COVID-19, The Journal of infection.

5. Lai, C. C., Shih, T. P., Ko, W. C., Tang, H. J. \& Hsueh, P. R. (2020) Severe acute respiratory syndrome coronavirus 2 (SARS-CoV-2) and coronavirus disease-2019 (COVID-19): The epidemic and the challenges, International journal of antimicrobial agents. 55, 105924.

6. (2020) [An update on the epidemiological characteristics of novel coronavirus pneumoniaCOVID19], Zhonghua liu xing bing xue za zhi = Zhonghua liuxingbingxue zazhi. 41, 139-144.

7. Anderson RM, H. H., Klinkenberg D, Hollingsworth TD (2020) in How will country-based mitigation measures influence the course of the COVID-19 epidemic pp. 931-934, The Lancet,

8. Jitendra Subhash, R., Aroni, C., Abhijeet, K. \& Shashikant, R. (2020) Targeting SARS-CoV-2 Spike Protein of COVID-19 with Naturally Occurring Phytochemicals: An in Silco Study for Drug Development.

9. Leng, Z., Zhu, R., Hou, W., Feng, Y., Yang, Y., Han, Q., Shan, G., Meng, F., Du, D., Wang, S., Fan, J., Wang, W., Deng, L., Shi, H., Li, H., Hu, Z., Zhang, F., Gao, J., Liu, H., Li, X., Zhao, Y., Yin, K., He, X., Gao, Z., Wang, Y., Yang, B., Jin, R., Stambler, I., Lim, L. W., Su, H., Moskalev, A., Cano, A., Chakrabarti, S., Min, K. J., Ellison-Hughes, G., Caruso, C., Jin, K. \& Zhao, R. C. (2020) Transplantation of ACE2(-) Mesenchymal Stem Cells Improves the Outcome of Patients with COVID-19 Pneumonia, Aging and disease. 11, 216-228.

10. Lu, R., Zhao, X., Li, J., Niu, P., Yang, B., Wu, H., Wang, W., Song, H., Huang, B., Zhu, N., Bi, Y., Ma, X., Zhan, F., Wang, L., Hu, T., Zhou, H., Hu, Z., Zhou, W., Zhao, L., Chen, J., Meng, Y., Wang, J., Lin, Y., Yuan, J., Xie, Z., Ma, J., Liu, W. J., Wang, D., Xu, W., Holmes, E. C., Gao, G. F., Wu, G., Chen, W., Shi, W. \& Tan, W. (2020) Genomic characterisation and epidemiology of 2019 novel coronavirus: implications for virus origins and receptor binding, Lancet. 395, 565-574.

11. Zhou, P., Yang, X. L., Wang, X. G., Hu, B., Zhang, L., Zhang, W., Si, H. R., Zhu, Y., Li, B., Huang, C. L., Chen, H. D., Chen, J., Luo, Y., Guo, H., Jiang, R. D., Liu, M. Q., Chen, Y., Shen, X. R., Wang, X., Zheng, X. S., Zhao, K., Chen, Q. J., Deng, F., Liu, L. L., Yan, B., Zhan, F. X., Wang, Y. 
Y., Xiao, G. F. \& Shi, Z. L. (2020) A pneumonia outbreak associated with a new coronavirus of probable bat origin, Nature. 579, 270-273.

12. Hoffmann, M., Kleine-Weber, H., Krüger, N., Müller, M., Drosten, C. \& Pöhlmann, S. (2020) The novel coronavirus 2019 (2019-nCoV) uses the SARS-coronavirus receptor ACE2 and the cellular protease TMPRSS2 for entry into target cells, bioRxiv, 2020.01.31.929042.

13. Golchin, A. \& Farahany, T. Z. (2019) Biological Products: Cellular Therapy and FDA Approved Products, Stem cell reviews and reports. 15, 166-175.

14. Golchin, A., Farahany, T. Z., Khojasteh, A., Soleimanifar, F. \& Ardeshirylajimi, A. (2019) The Clinical Trials of Mesenchymal Stem Cell Therapy in Skin Diseases: An Update and Concise Review, Current stem cell research \& therapy. 14, 22-33.

15. Bing Liang, J. C., Tao Li3, Haiying Wu4, Wenjie Yang1, Yanjiao Li5, Jianchun, Li, C. Y., Fangang Nie, Zhaoxia Ma, Mingxi Yang, Panrong Nie, Yanfeng Gao, \& Chuanyun Qian, M. H. (2020) Clinical remission of a critically ill COVID-19 patient treated by

human umbilical cord mesenchymal stem cells.

16. Glenn, J. D. \& Whartenby, K. A. (2014) Mesenchymal stem cells: Emerging mechanisms of immunomodulation and therapy, World journal of stem cells. 6, 526-39. 